\title{
Resveratrol Induces the Osteogenic Differentiation via MiR-320c by Targeting Runx2 and Is Involved in the Adipogenic differentiation of BMSCs
}

\section{Jilong Zou}

First Affiliated Hospital of Harbin Medical University

Jianyang Du

Second Affiliated Hospital of Harbin Medical University

Hualei Tu

Fifth Hospital of Harbin

\section{Hongjun Chen}

First Affiliated Hospital of Harbin Medical University

\section{Kai Cong}

First Affiliated Hospital of Harbin Medical University

\section{Zhenggang Bi}

First Affiliated Hospital of Harbin Medical University

Jiabing Sun ( $\nabla$ sunjiabing@live.cn )

First Affiliated Hospital of Harbin Medical University

\section{Research}

Keywords: Resveratrol, Bone mesenchymal stem cells, MicroRNAs, Osteogenic differentiation, Adipogenic differentiation

Posted Date: May 8th, 2020

DOI: https://doi.org/10.21203/rs.3.rs-25665/v1

License: (c) (1) This work is licensed under a Creative Commons Attribution 4.0 International License. Read Full License 


\section{Abstract \\ Background}

Bone marrow mesenchymal stem cells (BMSCs) are multipotent progenitor cells and have been widely used in clinical therapies due to their multiple pluripotency. Recent publications have found that resveratrol (RSVL) could promote the proliferation and differentiation of mesenchymal stem cells; however, the underlying molecular mechanism of RSVL-induced BMSCs osteogenic differentiation needs to be fully elucidated. The aim of this study was to investigate the function of miRNAs in RSVL-treated BMSCs and its effects on the osteogenic differentiation of BMSCs.

\section{Methods}

BMSCs were cultured and treated with different concentrations of RSVL. After osteogenic differentiation for 20 days, ALP staining was performed to evaluate the ALP activity of BMSCs. And ARS staining was used to detect the matrix mineralization deposition of BMSCs. After adipogenic differentiation for 20 days, adipogenic differentiation was determined by ORO staining for lipid droplets. Quantitative real-time polymerase chain reaction analysis was performed to assess the expression level of target genes. Bioinformatics analysis and luciferase reporter assay was ultilized to examine the relationship between miR-320c and its target gene. Western blot assay was used to analyze the protein expression level of target gene.

\section{Results}

Our results demonstrated that RSVL could promote the osteogenic differentiation and suppressed the adipogenic differentiation of BMSCs in a dose-dependent manner. Besides, a novel regulatory axis containing miR-320c and its target Runx2 was found during the differentiation process of BMSCs under RSVL treatment. Overexpression of miR-320c inhibited the osteogenic differentiation, while knockdown of miR-320c promoted the osteogenic differentiation of BMSCs. In contrast, overexpression of miR-320c accelerated the adipogenic differentiation, while knockdown of miR-320c restrained the adipogenic differentiation of BMSCs. Our results confirm that Runx2 was the directly target of miR-320c in RSVLpromoted osteogenic differentiation of BMSCs.

\section{Conclusions}

The present study revealed that miR-320c might possess the potentials as a novel clinical target for medical intervention to regulate the biological functions of RSVL in BMSCs.

\section{Introduction}


Bone marrow mesenchymal stem cells (BMSCs), derived from bone marrow, are multipotent progenitor cells and have the capability to differentiate into multiple lineages[1-3]. BMSCs undergo differentiation into various cell types, such as osteoblasts, adipocytes, fibroblasts and chondrocytes[4]. Due to their pronounced differentiation potentials, BMSCs have been widely used in clinical therapies, especially in bone regeneration and tissue engineering[5]. The osteogenic differentiation of BMSCs exhibits a vital role in the balance of bone environment[6]. A decreased number of osteoblasts might lead to the bone loss and even bone diseases, such as osteoporosis[1]. The osteogenic differentiation of BMSCs can be regulated by various signal pathways, but how to promote the osteogenic differentiation effectively is still a knotty problem.

In recent studies, a few researchers focused on the naturally occurring polyphenolic compound, resveratrol (3,4,5-trihydroxystilbene) (RSVL), which was found in red wine and some Asian medicinal herbs[7]. It has been reported that RSVL exhibits the anti-oxidation, anti- inflammatory anti-aging and anticancer functions[8-10]. Besides, previous studies showed that RSVL could attenuate the bone loss in ovariectomy-induced osteoporosis and promote the proliferation and osteogenic differentiation of mesenchymal stem cells[11-13]. However, the underlying molecular mechanism of RSVL-induced BMSCs osteogenic differentiation needs further investigation.

MicroRNAs (miRNAs) are a type of small regulatory noncoding RNA molecules at an approximate length of 22 nucleotides that function as negative regulators of gene expression via mRNA degradation or translational inhibition by directly binding to the 3 '-UTR of the gene mRNA[14, 15]. Accumulating evidence suggests that miRNAs play important roles in many biological processes, such as immune response, hematopoiesis, tumorigenesis, tissue differentiation and organogenesis[2, 16]. In recent publications, abnormal expression of miRNAs has been found to be involved in the bone regeneration and bone tissue engineering[17]. However, the effects of miRNAs on RSVL-induced BMSCs osteogenic differentiation have not been investigated.

In the present study, we investigated the functional roles of RSVL and miR-320c in the osteogenic differentiation of BMSCs in vitro, and explored whether RSVL regulated this biological process through the miR-320c/ runt-related transcription factor 2 (Runx2) network. The results indicated that RSVL could promote the osteogenic differentiation of BMSCs in a dose-dependent manner and found that miR-320c was downregulated in RSVL-treated BMSCs. Further researches uncovered that miR-320c regulated the RSVL-induced BMSCs osteogenic differentiation and adipogenic differentiation of BMSCs by directly targeting runt-related transcription factor 2 (Runx2). Thus, the current study supported that miR$320 \mathrm{c} /$ Runx 2 might be an ideal predictor and therapeutic candidate for treating bone loss. Our findings might facilitate the development of new therapeutic strategies against bone diseases, such as osteoporosis.

\section{Materials And Methods}

\section{Reagents}


RSVL was purchased from Must Bio-Technology Co., Ltd. (Chengdu, China) and its purity was more than 99\% confirmed by high-performance liquid chromatography (HPLC). RSVL was dissolved in dimethyl sulfoxide (DMSO) (Biotopped, Beijing, China) and the final culture concentration of DMSO was less than 0.5\%. Dulbecco's modified Eagle's medium (DMEM) was purchased from HyClone (Logan, UT, USA). Fetal bovine serum (FBS) was provided by Roya Bio-Technology Co., Ltd (Lanzhou, China). Alizarin red S (ARS) staining kit was purchased from Sigma (St. Louis, MO, USA). The miR-320c mimics, miR-320c inhibitor and negative controls (NC) were obtained from GenePharma (Jiangsu, China). Lipofectamine 2000 was purchased from Invitrogen (Carlsbad, CA, USA). Anti-Runx2 and anti-bone sialoprotein (BSP) antibodies were purchased from Biosynthesis biotechnology Co., Ltd. (Beijing, China). Anti-osteocalcin (OCN) and anti-glyceraldehyde-3-phosphate dehydrogenase (GAPDH) antibodies were obtained from Protein tech (Wuhan, China). Anti-PPARy and anti-FABP4 were purchased from Cell Signalling Technology, USA.

\section{Cell Culture}

Human BMSCs were purchased from Cyagen, China. The cell culture was performed as previous described [18]. In brief, human BMSCs were cultured using DMEM medium supplemented with $15 \%$ FBS, $100 \mathrm{U} / \mathrm{ml}$ penicillin and $1 \%$ streptomycin. Then the medium containing BMSCs was seeded into $25 \mathrm{~cm}^{2}$ flasks and the culture medium was replaced every 3 days. Non-adherent cells were discarded during the medium replacement and BMSCs were harvested as a result of their adherence characteristic.

\section{Osteogenic Differentiation}

For purposes of osteogenic differentiation, the BMSCs were seeded in 24-well plates at a density of $5 \times$ $10^{5} \mathrm{cells} / \mathrm{ml}$ and cultured in the osteogenic differentiation medium containing DMEM medium, $15 \% \mathrm{FBS}$, $10 \mathrm{nM}$ dexamethasone, $50 \mathrm{mg} / \mathrm{L}$ ascorbic acid and $10 \mathrm{mM} \beta$-glycerophosphate. RSVL $(0,2,4$ or $8 \mu \mathrm{M})$ was added to the osteogenic differentiation medium, which was replaced every 3 days. The cells were cultured in the osteogenic differentiation medium for 20 days and used for further analysis.

\section{Alkaline Phosphatase (ALP) Staining}

After exposure to different treatments, ALP staining was performed to evaluate the ALP activity of BMSCs. The assay was conducted according to the manufacturer's recommended protocol. Briefly, BMSCs were gently washed twice with PBS and then fixed with $95 \%$ ethanol for 10 min at $37^{\circ} \mathrm{C}$. After washing three times again, the cells were incubated with staining solution containing $2 \%$ magnesium sulfate, $2 \%$ calcium chloride, $3 \%$-glycerophosphate disodium salt hydrate and $2 \%$ sodium pentobarbital for $4 \mathrm{~h}$ at room temperature. In the last $5 \mathrm{~min}, 2 \%$ cobalt nitrate was added. Then, the cells were washed twice with PBS and incubated with 1\% ammonium sulfide for another 2 min. Finally, the stained cells were washed with double distilled water and photographed using a light microscopy (Nikon, Japan). 
Image Pro Plus 6.0 (Media Cybernetics, Rockville, MD, USA) was used to qualified the assay and each experiment was performed in triplicate.

\section{Alizarin Red S (ARS) Staining}

ARS staining was used to detect the matrix mineralization deposition of BMSCs suffering different treatments. Briefly, the cells were washed twice with phosphate buffered saline (PBS), followed by fixation with $4 \%$ paraformaldehyde $(\mathrm{m} / \mathrm{v})$ for $10 \mathrm{~min}$ at room temperature. After washing three times again with PBS, the cells were stained with $40 \mathrm{mM}$ ARS (pH 4.2) for 15 minutes. Finally, the stained cells were washed with double distilled water and photographed using a light microscopy (Nikon, Japan). To quantify the assay, the stained cells were incubated with $10 \%$ cetylpyridinium chloride for 20 min at $37^{\circ} \mathrm{C}$. Then, the supernatant was collected and measured at a wavelength of $562 \mathrm{~nm}$ using a fluorescence spectrophotometer (BioTek, USA). The assay was repeated at least three times independently.

\section{Adipogenic Differentiation}

To induce adipogenic differentiation of BMSCs in vitro, the cells were cultured in 24-well plates. And the adipogenic differentiation medium containing $0.5 \mathrm{mM}$ isobutylmethylxanthine, $0.5 \mu \mathrm{M}$ dexamethasone, $10 \mu \mathrm{g} / \mathrm{mL}$ insulin and $60 \mu \mathrm{M}$ indomethacin (Sigma-Aldrich, USA), was applied when the cells reached $95 \%$ confluence. The induction medium was changed every third day and the cells were induced for 20 days.

\section{Oil Red O (ORO) Staining}

After adipogenic differentiation for 20 days, adipogenic differentiation was determined by ORO staining for lipid droplets. The cells were rinsed by PBS and then fixed with 4\% paraformaldehyde for $30 \mathrm{~min}$ at room temperature. After that, the cells were stained with ORO staining solution (Cyagen, China) for $40 \mathrm{~min}$ at room temperature. Finally, the mineralized extracellular matrix was observed and photographed under the inverted optical microscope (Nikon, Japan). And the number of lipid droplets was analyzed.

\section{Transfection Of Oligonucleotides}

To confirm the function of miR-320c, miR-320c mimics, miR-320c inhibitor and negative controls (NCs) (GenePharma, Jiangsu, China) were transfected into BMSCs using Lipofectamine 2000 (Invitrogen, Carlsbad, CA, USA) according to the manufacturer's instructions. The final concentration of miR-320c mimics was $50 \mathrm{nM}$, while the final concentration of miR-320c mimics was $100 \mathrm{nM}$. After incubation for 20 days, the cells were used to perform ARS, ALP and ORO stainings or additional analysis. 
Total RNA extraction and quantitative real-time polymerase chain reaction (real time qPCR)

After exposure to different treatments, BMSCs were washed twice with cold PBS and total RNA was isolated using TRIzol reagents (Invitrogen, Carlsbad, CA, USA) following the manufacturer's protocol. Then, the cDNAs were synthesized from $0.5 \mu \mathrm{g}$ extracted total RNAs using the High-Capacity cDNA Reverse Transcription Kits (Applied Biosystems, Foster City, CA, USA) and real time qPCR was implemented with ABI 7500 real-time PCR system (Applied Biosystems) as described previously [19]. The real time qPCR program was as follows: initial denaturation at $50^{\circ} \mathrm{C}$ for 2 min and $95^{\circ} \mathrm{C}$ for $4 \mathrm{~min}$, followed by 40 cycles of $90^{\circ} \mathrm{C}$ for $30 \mathrm{~s}$ and $60^{\circ} \mathrm{C}$ for $30 \mathrm{~s}$. The expression of target genes was normalized by the $2^{-\triangle \triangle C t}$ method. The house-keeping genes $U 6$ and GAPDH were used as controls. Each result of real time qPCR was repeated three times independently.

\section{Plasmid Constructions And Luciferase Reporter Assay}

A fragment of RunX2 3'-UTR containing the predicted binding sequence for miR-320c was inserted into the pGL6-miR vector (Beyotime Biotechnology, Shanghai, China) to construct a wild-type Runx2-3'UTR luciferase reporter gene plasmid (Runx2-3'UTR-WT) and the mutant type (Runx2-3'UTR-MUT) was generated via the similar procedure except for site-directed mutagenesis of the target sequence. For luciferase reporter assay, HEK293 cells were seeded in 96-well plate and co-transfected with miR-320c mimics, negative controls, Runx2-3'UTR-WT or Runx2-3'UTR-MUT using Lipofectamine 2000 (Invitrogen, Carlsbad, CA, USA) according to the manufacturer's recommended instructions. After $48 \mathrm{~h}$ incubation, cells were lysed and luciferase activity was detected using the Dual-Luciferase Reporter Assay System (Promega Corporation, Madison, WI, USA). Renilla-luciferase was used as internal control.

\section{Western Blot Analysis}

Western blot was performed following the protocol of previous reports [20]. In brief, BMSCs were lysed with RIPA buffer containing $1 \%$ phenylmethylsulfonyl fluoride and centrifuged at 13,500 rpm for $15 \mathrm{~min}$ at $4{ }^{\circ} \mathrm{C}$. The protein concentrations were confirmed using a BCA Protein Assay Kit (Beyotime Biotechnology, Shanghai, China). Then equal amounts $(60 \mu \mathrm{g})$ of protein were applied to $12-15 \%$ sodium dodecyl sulphate-polyacrylamide gel electrophoresis and transferred to nitrocellulose membranes. After blocked in $5 \%$ nonfat milk for $1 \mathrm{~h}$, the membranes were incubated with primary antibodies at $4{ }^{\circ} \mathrm{C}$ overnight and secondary antibodies for $1 \mathrm{~h}$ at room temperature. Finally, the immunoreactive bands were obtained and quantified by using the Odyssey Infrared Imaging System (LI-COR Biotechnology, Nebraska, USA).

\section{Bioinformatics Analysis}


GSE74209 was applied for the further analysis to detect the underlying mechanism at the level of miRNAs in the Gene Expression Omnibus (GEO) database. The "Limma" R package was used to screen expression data for differentially expressed genes (DEGs) between healthy controls and osteoporotic samples[21]. The selection criteria were as follows: false discovery rate $(F D R)<0.05$, and $\|$ log 2 fold change| $>2$. The values for genes with the same names were averaged.

\section{Statistical analysis}

The data were presented as the mean \pm SEM. Statistical analysis was conducted using Student's t-test for comparing the differences between two groups. One-way ANOVA method was used for the comparisons of multiple groups. All experiments were repeated at least three times, and representative experiments were shown. $p<0.05$ was considered statistically significant.

\section{Results}

\section{RSVL promotes the osteogenic differentiation of BMSCs}

To confirm whether RSVL could promote the osteogenic differentiation of BMSCs, we treated them with different concentrations of $\operatorname{RSVL}(0,2,4$ and $8 \mu \mathrm{M})$ under osteogenic induction for 20 days. First, the structure of RSVL was shown in Fig. 1A. Then, ALP and ARS stainings were applied to detect the roles of RSVL in the osteogenic differentiation of BMSCs. ALP staining showed that, with the increase in the concentration of RSVL, the osteogenic differentiation of BMSCs gradually increased, suggesting that RSVL significantly promoted the osteogenesis of BMSCs in a dose-dependent manner (Fig. 1B). ARS staining and quantification revealed that extracellular matrix mineralization was observably increased by RSVL treatment at 20 days after osteogenic differentiation (Fig. 1C). As shown in supporting Fig. 1D, RSVL treatment significantly upregulated the expressions of osteoblast-related genes, including bone morphogenetic protein (BMP4), Osterix, osteopontin (OPN) and Collagen type I (Col1A), in a dosedependent manner when added RSVL at concentrations of 2, 4 and $8 \mu \mathrm{M}$ (Fig. 1D). To further test the potential roles of RSVL in osteogenic differentiation, BMSCs with or without RSVL treatment were cultured in osteogenic induction medium for 20 days, and the protein expression levels of osteogenesisassociated genes, including osteocalcin (OCN) and bone sialoprotein (BSP) were detected by western blot analysis. As shown in Fig. 1E, the protein expression levels of OCN and BSP were found to be increased significantly in cells treated with RSVL (Fig. 1E). Besides, with the increased concentrations of RSVL, the level of OCN and BSP gradually upregulated (Fig. 1E). Taken together, these results demonstrated that RSVL markedly facilitated the osteogenesis of BMSCs in a dose-dependent manner.

\section{miR-320c was downregulated during osteogenic differentiation of BMSCs and under the treatment of RSVL}

Previous studies showed that miRNAs played an important role in regulating the osteogenic differentiation of BMSCs. GSE74209, acted as a microRNA array, was applied for the further analysis to 
detect the expression of dysregulated genes in the Gene Expression Omnibus (GEO) database. As shown in Fig. 2A, miR-320c exhibited the upregulated expression according to the results of the differential analysis (Fig. 2A). The heatmap showed that miR-320c was significantly upregulated in the samples from osteoporotic patients, suggesting that miR-320c might be related to the occurrence and development of bone diseases characterized by bone loss, such as osteoporosis (Fig. 2B). By screening the aberrantly expressed miRNAs during osteogenic differentiation. BMSCs were induced into osteoblasts for $0,4,8,12,16$ and 20 days (Fig. 2C and 2D). Real time qPCR analysis indicated that the expression level of miR-320c decreased along with the osteogenic induction for different time, and the expression level of miR-320c was the lowest after osteogenesis for 20 days, suggesting that miR-320c expression was reduced in a time-dependent manner (Fig. 2E). Then, we subsequently detected the expression level of miR-320c using real time qPCR in RSVL-treated BMSCs. As shown in Fig. 2F, miR-320c expression was reduced in a dose-dependent manner under RSVL treatment, which revealed that miR-320c might participate in the regulation of RSVL-promoted osteogenic differentiation of BMSCs.

\section{The roles of miR-320c in the osteogenic differentiation of BMSCs}

To evaluate the effects of miR-320c in the osteogenesis of BMSCs in vivo, the BMSCs transfected with miR-320c mimics or NC were subjected to osteogenic induction for 20 days. ALP staining showed that compared with NC, miR-320c mimics significantly decreased the osteogenic differentiation of BMSCs, suggesting that over-expression of miR-320c in BMSCs could lead to the decreased osteogenic differentiation of BMSCs (Fig. 3A). ARS staining also indicated that transfection of miR-320c mimics markedly suppressed the osteogenic differentiation of BMSCs (Fig. 3B). Further, real time qPCR analysis revealed significantly lower expression of bone matrix genes, including BMP4, Osterix, OPN and Col1A, in miR-320c mimics transfected BMSCs than in NC transfected BMSCs (Fig. 3C). The effects of miR-320c inhibitor were then evaluated in the osteogenic differentiation of BMSCs. Interestingly, ALP and ARS stainings exhibited that the osteogenesis was markedly higher in miR-320c inhibitor group than in NC group, suggesting that knockdown of miR-320c in BMSCs could result in the increased osteogenic differentiation (Fig. 3D and 3E). Real time qPCR analysis revealed the mRNA expression levels of markers of osteogenesis (BMP4, Osterix, OPN and Col1A) were increased in BMSCs after transfection of miR-320c inhibitor (Fig. 3F). These results demonstrate that overexpression of miR-320c inhibited the osteogenic differentiation, while knockdown of miR-320c promoted the osteogenic differentiation of BMSCs.

\section{The effects of RSVL on the adipogenic differentiation of BMSCs and expression of miR-320c during the adipogenic differentiation of BMSCs}

To investigate the roles of RSVL in the adipogenic differentiation, we treated BMSCs with RSVL at different concentrations and induced them into adipocytes. ORO staining indicated that the number of oil droplets decreased after treatment of RSVL in a manner-dependent manner, indicating that RSVL might play a negative role in the adipogenesis of BMSCs (Fig. 4A). Besides, compared with the control group, 2 , 4 and $8 \mu \mathrm{M}$ RSVL decreased the mRNA expression levels of adipogenesis-related genes, including leptin, lipoprotein lipase (LPL), adiponectin, and adipocyte protein $2(a \mathrm{P} 2)$ in different degrees (Fig. 4B). As 
shown in the results of western blot, different concentrations of RSVL significantly downregulated the protein expression levels of peroxisome proliferator-activated receptor gamma (PPARY) and fatty acid binding protein 4 (FABP4), important markers of adipogenesis (Fig. 4C). To further detect the effects of miR-320c on the adipogenic differentiation of BMSCs, the BMSCs were induced into adipocytes under adipogenic differentiation medium. The BMSCs were respectively cultured in adipogenic differentiation medium for $0,4,8,12,16$ and 20 days (Fig. 4D). ORO staining indicated that the number of lipid oil droplets significantly increased with the extension of time (Fig. 4D and 4E). Besides, miR-320c expression was revealed by real time qPCR analysis to gradually increase during adipogenic differentiation of BMSCs, peaking at the adipogenic differentiation of 20 days (Fig. 4F). The above results showed that RSVL significantly inhibited the adipogenic differentiation of BMSCs and miR-320c was upregulated during adipogenesis of BMSCs.

\section{The effects of miR-320c on the adipogenic differentiation of BMSCs}

To overexpress or silence miR-320c in BMSCs for functional investigation, we transfected BMSCs with miR-320c mimics, miR-320c inhibitor or NCs and subsequently cultured in adipogenic differentiation medium. As shown in Fig. 5A, ORO staining revealed that overexpression of miR-320c increased the lipid droplet formation in adipogenesis-induced BMSCs, compared with NC (Fig. 5A). Likewise, the mRNA expression levels of four important adipocyte-specific markers, leptin, LPL, adiponectin, and $a \mathrm{P} 2$, were also up-regulated compared with NC (Fig. 5B). Conversely, knockdown of miR-320c markedly attenuated the lipid droplet formation of BMSCs under adipogenic differentiation induction, dected by ORO staining (Fig. 5C). In addition, silence of miR-320c suppressed the expression level of adipocyte-related genes, including leptin, $\mathrm{LPL}$, adiponectin, and $a \mathrm{P} 2$, of BMSCs after adipogenic differentiation (Fig. 5D).

\section{Overexpression of miR-320c reversed the increased osteogenic differentiation and decreased adipogenic differentiation of BMSCs caused by RSVL}

To investigate the functions of miR-320c in RSVL-promoted BMSCs osteogenic differentiation, we used miR-320c mimics to overexpress miR-320c in BMSCs after treatment of $8 \mu \mathrm{M} R S V L$. The results of ALP staining showed that overexpression of miR-320c significantly reduced the matrix mineralization deposition and ALP activity, which was upregulated by $8 \mu \mathrm{M}$ RSVL treatment in BMSCs under osteogenic induction (Fig. 6A). Meanwhile, ARS staining also indicated that in the presence of miR-320c mimics, the osteogenic differentiation increased by $8 \mu \mathrm{M}$ RSVL treatment was significantly decreased (Fig. 6B). Besides, the upregulated mRNA expression level of osteoclast-specific genes in the RSVL-treated BMSCs were significantly down-regulated, including leptin, LPL, adiponectin, and AP2 (Fig. 6C). To further investigate the role of miR-320c in RSVL-suppressed BMSCs adipogenic differentiation, BMSCs were transfected with miR-320c mimics and treated with RSVL under adipogenic differentiation medium. ORO staining revealed that the number of oil droplets were decreased by the RSVL treatment, which could be increased by overexpression of miR-320c (Fig. 6D). Real time qPCR analysis indicated that the mRNA expression level of adipocyte-specific genes, including leptin, $\mathrm{LPL}$, adiponectin, and $a \mathrm{P} 2$, was reduced by RSVL treatment and then downturned by miR-320c mimics (Fig. 6E). The above data suggested that miR- 
320c played a negative role in RSVL-promoted osteogenic differentiation and RSVL-suppressed adipogenic differentiation of BMSCs.

\section{MiR-320c regulates RSVL-promoted osteogenic differentiation of BMSCs by targeting Runx2}

To confirm the possible targets of miR-320c in RSVL-promoted BMSCs osteogenic differentiation, two miRNA target prediction systems (TargetScan Human 7.2) were used in our study to look for miR-320ctargeting mRNAs. Among the putative mRNAs, we noticed that Runx2 had close relationship with miR$320 \mathrm{c}$ and the binding sites between miR-320 c and Runx2 were predicted using miRanda and TargetScan (Fig. 7A). Based on these data, we speculated that Runx2 was the directly target of miR-320c. Luciferase reporter assay was performed to verify the hypothesis. Luciferase reporter assay showed that the relative luciferase activity of Runx2-3'UTR-WT was significantly repressed duo to miR-320c mimics' addition when compared with control (Fig. 7B). In contrast, the predicted binding sequence mutation completely abrogated this repression (Fig. 7B). Additionally, real time qPCR and western blot analysis revealed that overexpression of miR-320c could decrease the Runx2 expression, while knockdown of miR-320c could increase the Runx2 expression in BMSCs (Fig. 7C-7E). Taken together, these results confirm that Runx2 was the directly target of miR-320c in RSVL-promoted osteogenic differentiation of BMSCs.

\section{Discussion}

BMSCs are multipotent progenitor cells and have been wildly used in clinical therapies due to their differentiation potentials[22, 23]. Recent publications reported that RSVL could promote the proliferation and differentiation of mesenchymal stem cells, however, the underlying molecular mechanism of RSVLinduced BMSCs osteogenic differentiation is still unclear. In the present study, we demonstrated that RSVL could dose-dependently promote the osteogenic differentiation of BMSCs and miR-320c played an important role in regulating the differentiation process by directly targeting Runx2. Our work showed for the first time that miRNAs could modulate RSVL-induced BMSCs osteogenic differentiation and provided a novel perspective for the study of RSVL's biological functions.

RSVL, a naturally occurring non-flavonoid polyphenol, was reported to possess multiple health benefits[24, 25]. It can prevent the occurrence of cardiovascular diseases and alleviate the symptoms of the existing disorders, such as cancer, diabetes and obesity[26-30]. Our results showed that RSVL could promote the osteogenic differentiation of BMSCs, which was consistent with the previous publications. Nevertheless, because of its structural similarity to estrogen, most scholars paid their attention to estrogen-related mechanism. Other mechanisms have rarely been studied. In the current study, we focused on the aberrantly expressed miRNAs in RSVL-induced osteogenic differentiation of BMSCs to explore its molecular mechanism.

In recent years, numerous studies deciphered the functions of miRNAs in regulating BMSCs differentiation. MiR-31 was reported to act as a negative regulator in osteoblastic differentiation of BMSCs[31]. Inhibiting miR-31 could enhance the matrix mineralization deposition and ALP activity of BMSCs via increasing the expression of BSP, Osterix and osteopontin (OPN)[32]. MiR-205 repressed the 
osteoblastic differentiation of BMSCs in virtue of reducing the expression of SATB2 and Runx2[33]. MiR$320 \mathrm{c}$ was previously found to be involved in some different malignancies, such as pancreatic cancer, hepatocellular carcinoma and bladder cancer[34]. A recent study revealed that miR-320c could regulate adipocyte differentiation of human mesenchymal stem cells, whereas its function in osteoblastic differentiation of BMSCs has not been reported in detail[35]. In the present study, we demonstrated that miR-320c was downregulated and functioned as a negative regulator during RSVL-promoted osteogenic differentiation of BMSCs. To get a better understanding of the mechanism, we used miRanda and TargetScan to predict its possible targets and identified our speculation by means of luciferase assay. Our results showed that miR-320c could directly interact with Runx2' $3^{\prime}$-UTR and subsequently inhibit its expression, which revealed that miR-320c regulated BMSCs osteogenic differentiation through targeting Runx2.

Runx 2 is a crucial transcriptional regulator of bone formation and plays an important role in modulating the differentiation of mesenchymal stem cells[36-38]. Previous studies showed that Runx2 was the target of many miRNAs, such as miR-23a, miR-30c, miR-204 and miR-143-5p [39-41]. That is not surprising because Runx2 has a long 3'-UTR ( 3777 b) which makes it more likely to be the target of miRNAs, including miR-320c in this study. Our results identified that Runx2 was the effector of miR-320c and exerted important influences on RSVL-promoted osteogenic differentiation of BMSCs. By targeting Runx2, miR-320c indirectly regulated the expression of osteogenic markers, such as OCN and BSP, thereby participated in the modulation of RSVL-promoted BMSCs differentiation.

In conclusion, our study revealed that RSVL could promote the osteogenic differentiation but inhibited the adipogenic differentiation of BMSCs under induction condition. Besides, a novel regulatory axis containing miR-320c and its target Runx2 was found during the differentiation process. As far as we know, this is the first research to elucidate the function of miRNAs in RSVL-promoted BMSCs differentiation in detail. Additionally, our findings suggested that miR-320c could be a target for medical intervention to control the biological effects of RSVL on BMSCs.

\section{Abbreviations}

BMSCs: bone marrow mesenchymal stem cells; RSVL: resveratrol; miRNAs: microRNAs; Runx2: runtrelated transcription factor 2; HPLC: high-performance liquid chromatography; DMSO: dimethyl sulfoxide; DMEM: Dulbecco's modified Eagle's medium; FBS: fetal bovine serum; ARS: alizarin red S; NC: negative controls; BSP: bone sialoprotein; OCN: osteocalcin; GAPDH: glyceraldehyde-3-phosphate dehydrogenase; DEGs: differentially expressed genes; Col1A: Collagen type I; GEO: Gene Expression Omnibus; LPL: lipoprotein lipase; $a \mathrm{P} 2$ : adipocyte protein 2; PPARY: peroxisome proliferator-activated receptor gamma; FABP4: fatty acid binding protein 4; OPN: osteopontin.

\section{Declarations}

\section{Acknowledgements}


Not applicable.

\section{Authors' contributions}

Jiabing Sun and Jilong Zou conceived the project and finished the manuscript. Jilong Zou performed the cell culture, miRNA transfection, ALP staining, ARS stainings and ORO stainings. Jianyang Du conducted the bioinformatics analysis and statistical analysis. Hualei Tu and Hongjun Chen performed the RNA isolation and real time qPCR analysis and western blot analysis. Kai Cong and Zhenggang Bi modified the manuscript and provided the technique support.

\section{Funding}

The study was supported by the Natural Science Foundation of Heilongjiang (Grant No. H2015064), the Innovation Foundation of the First Affiliated Hospital of Harbin Medical University (Grant No. 2019B15) and the Foundation of Heilongjiang Health Committee (Grant No. 2019-012).

\section{Availability of data and materials}

Not applicable.

\section{Ethics approval and consent to participate}

Not applicable.

\section{Consent for publication}

Not applicable.

\section{Competing interests}

The authors indicated no potential conflicts of interest.

\section{Author details}

${ }^{1}$ Department of Orthopaedics, the First Affiliated Hospital of Harbin Medical University, Harbin 150001, China. ${ }^{2}$ Department of Neurosurgery, the Second Affiliated Hospital of Harbin Medical University, Harbin, 150086, China ${ }^{3}$ Department of Burn, the Fifth Hospital in Harbin, Harbin 150036, China

\section{References}

1. Li CJ, et al. Long noncoding RNA Bmncr regulates mesenchymal stem cell fate during skeletal aging. J Clin Invest. 2018;128(12):5251-66.

2. Li KC, et al. Healing of osteoporotic bone defects by baculovirus-engineered bone marrow-derived MSCs expressing MicroRNA sponges. Biomaterials. 2016;74:155-66. 
3. Liao L, et al. Redundant let-7a suppresses the immunomodulatory properties of BMSCs by inhibiting the Fas/FasL system in osteoporosis. FASEB J. 2018;32(4):1982-92.

4. Luo ZW, et al. Aptamer-functionalized exosomes from bone marrow stromal cells target bone to promote bone regeneration. Nanoscale. 2019;11(43):20884-92.

5. Li Y, et al. miR-149-3p Regulates the Switch between Adipogenic and Osteogenic Differentiation of BMSCs by Targeting FTO. Mol Ther Nucleic Acids. 2019;17:590-600.

6. Tang Q, et al. Microporous polysaccharide multilayer coated BCP composite scaffolds with immobilised calcitriol promote osteoporotic bone regeneration both in vitro and in vivo. Theranostics. 2019;9(4):1125-43.

7. Jeandet $\mathrm{P}$, et al., Whole-cell biocatalytic, enzymatic and green chemistry methods for the production of resveratrol and its derivatives. Biotechnol Adv, 2019: p. 107461.

8. Zhou T, et al. Resveratrol improves osteogenic differentiation of senescent bone mesenchymal stem cells through inhibiting endogenous reactive oxygen species production via AMPK activation. Redox Rep. 2019;24(1):62-9.

9. Garcia-Quiroz J, et al., Synergistic Antitumorigenic Activity of Calcitriol with Curcumin or Resveratrol is Mediated by Angiogenesis Inhibition in Triple Negative Breast Cancer Xenografts. Cancers (Basel), 2019. 11(11).

10. Chaudhary Z, et al. Encapsulation and Controlled Release of Resveratrol Within Functionalized Mesoporous Silica Nanoparticles for Prostate Cancer Therapy. Front Bioeng Biotechnol. 2019;7:225.

11. Lv YJ, et al. Resveratrol counteracts bone loss via mitofilin-mediated osteogenic improvement of mesenchymal stem cells in senescence-accelerated mice. Theranostics. 2018;8(9):2387-406.

12. Domazetovic $\mathrm{V}$, et al. Protective role of benzoselenophene derivatives of resveratrol on the induced oxidative stress in intestinal myofibroblasts and osteocytes. Chem Biol Interact. 2017;275:13-21.

13. Zhao $L$, et al. Effects of dietary resveratrol on excess-iron-induced bone loss via antioxidative character. J Nutr Biochem. 2015;26(11):1174-82.

14. Inoue K, et al. Bone protection by inhibition of microRNA-182. Nat Commun. 2018;9(1):4108.

15. Song H, et al. Reversal of Osteoporotic Activity by Endothelial Cell-Secreted Bone Targeting and Biocompatible Exosomes. Nano Lett. 2019;19(5):3040-8.

16. Zhang X, et al. Cell-free 3D scaffold with two-stage delivery of miRNA-26a to regenerate critical-sized bone defects. Nat Commun. 2016;7:10376.

17. Liu J, et al. A delivery system specifically approaching bone resorption surfaces to facilitate therapeutic modulation of microRNAs in osteoclasts. Biomaterials. 2015;52:148-60.

18. Yang F, et al. Astragalus Polysaccharide Attenuated Iron Overload-Induced Dysfunction of Mesenchymal Stem Cells via Suppressing Mitochondrial ROS. Cell Physiol Biochem. 2016;39(4):1369-79.

19. Yang F, et al., Melatonin protects bone marrow mesenchymal stem cells against iron overloadinduced aberrant differentiation and senescence. J Pineal Res, 2017. 
20. Zou J, et al. Deoxyelephantopin Induces Reactive Oxygen Species-Mediated Apoptosis and Autophagy in Human Osteosarcoma Cells. Cell Physiol Biochem. 2017;42(5):1812-21.

21. Ritchie ME, et al. limma powers differential expression analyses for RNA-sequencing and microarray studies. Nucleic Acids Res. 2015;43(7):e47.

22. Wilkesmann S, et al., Primary osteoblasts, osteoblast precursor cells or osteoblast-like cell lines: which human cell types are (most) suitable for characterizing 45S5-Bioglass? J Biomed Mater Res A, 2019.

23. Cai $\mathrm{H}$, et al. Exosomal MicroRNA-9-3p Secreted from BMSCs Downregulates ESM1 to Suppress the Development of Bladder Cancer. Mol Ther Nucleic Acids. 2019;18:787-800.

24. Alkhalaf M, Jaffal S. Potent antiproliferative effects of resveratrol on human osteosarcoma SJSA1 cells: Novel cellular mechanisms involving the ERKs/p53 cascade. Free Radic Biol Med. 2006;41(2):318-25.

25. Osman AM, et al. Chemosensitizing and nephroprotective effect of resveratrol in cisplatin -treated animals. Cancer Cell Int. 2015;15:6.

26. Cao X, et al. Resveratrol prevents Angll-induced hypertension via AMPK activation and RhoA/ROCK suppression in mice. Hypertens Res. 2014;37(9):803-10.

27. Ji Q, et al. Resveratrol suppresses epithelial-to-mesenchymal transition in colorectal cancer through TGF-beta1/Smads signaling pathway mediated Snail/E-cadherin expression. BMC Cancer. 2015;15:97.

28. Timmers $\mathrm{S}$, et al. Calorie restriction-like effects of 30 days of resveratrol supplementation on energy metabolism and metabolic profile in obese humans. Cell Metab. 2011;14(5):612-22.

29. Ozturk E, et al. Resveratrol and diabetes: A critical review of clinical studies. Biomed Pharmacother. 2017;95:230-4.

30. Chakraborty S, Levenson AS, Biswas PK. Structural insights into Resveratrol's antagonist and partial agonist actions on estrogen receptor alpha. BMC Struct Biol. 2013;13:27.

31. Xie Q, et al. Effects of miR-31 on the osteogenesis of human mesenchymal stem cells. Biochem Biophys Res Commun. 2014;446(1):98-104.

32. Deng $Y$, et al. Effects of a miR-31, Runx2, and Satb2 regulatory loop on the osteogenic differentiation of bone mesenchymal stem cells. Stem Cells Dev. 2013;22(16):2278-86.

33. Hu N, et al. Regulative Effect of Mir-205 on Osteogenic Differentiation of Bone Mesenchymal Stem Cells (BMSCs): Possible Role of SATB2/Runx2 and ERK/MAPK Pathway. Int J Mol Sci. 2015;16(5):10491-506.

34. Iwagami Y, et al. miR-320c regulates gemcitabine-resistance in pancreatic cancer via SMARCC1. Br J Cancer. 2013;109(2):502-11.

35. Wang X, et al. MicroRNA-320c inhibits tumorous behaviors of bladder cancer by targeting Cyclindependent kinase 6. J Exp Clin Cancer Res. 2014;33:69. 
36. He, J., et al., In Vitro and < $i>$ In Vivo $</ i>$ biocompatibility study on acellular sheep periosteum for guided bone regeneration. Biomed Mater, 2019.

37. Colbath AC, et al., Can extracorporeal shockwave promote osteogenesis of equine bone marrowderived mesenchymal stem cells in vitro? Stem Cells Dev, 2019.

38. Wang Z, et al., Mg(2+) in beta-TCP/Mg-Zn composite enhances the differentiation of human bone marrow stromal cells into osteoblasts through MAPK-regulated Runx2/Osx. J Cell Physiol, 2019.

39. Hamam D, et al. microRNA-320/RUNX2 axis regulates adipocytic differentiation of human mesenchymal (skeletal) stem cells. Cell Death Dis. 2014;5:e1499.

40. Zhan FL, Liu XY, Wang XB. The Role of MicroRNA-143-5p in the Differentiation of Dental Pulp Stem Cells into Odontoblasts by Targeting Runx2 via the OPG/RANKL Signaling Pathway. J Cell Biochem. 2018;119(1):536-46.

41. Zhang $Y$, et al. A program of microRNAs controls osteogenic lineage progression by targeting transcription factor Runx2. Proc Natl Acad Sci U S A. 2011;108(24):9863-8.

\section{Figures}


A<smiles>Oc1ccc(/C=C/c2cc(O)cc(O)c2)cc1</smiles>

B

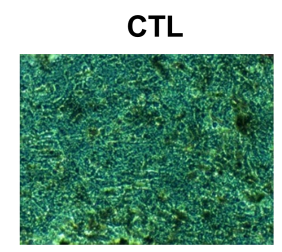

$\operatorname{RSVL}(2 \mu \mathrm{M})$

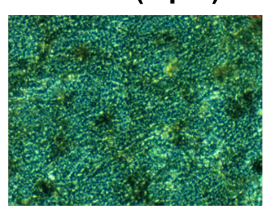

RSVL (4 $\mu \mathrm{M})$
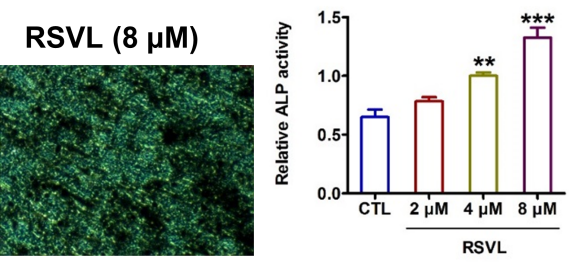

C

CTL
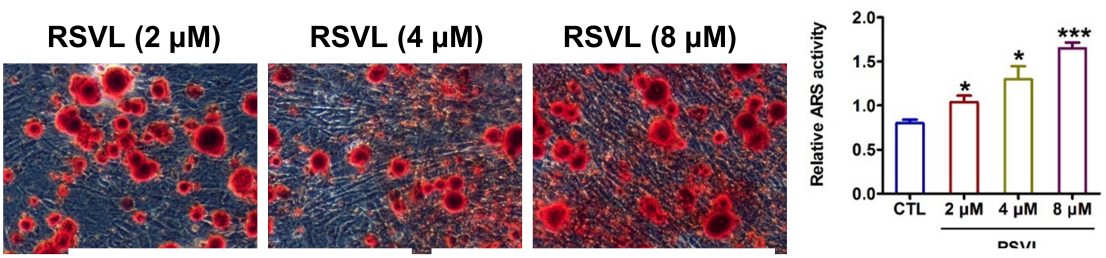

D
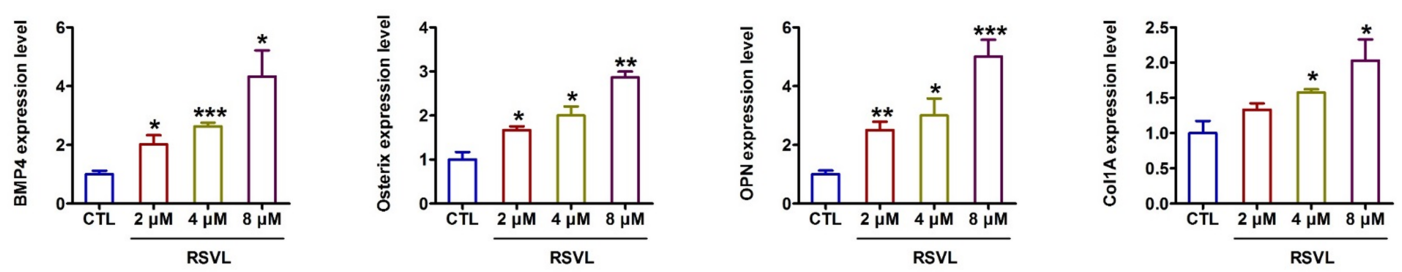

E
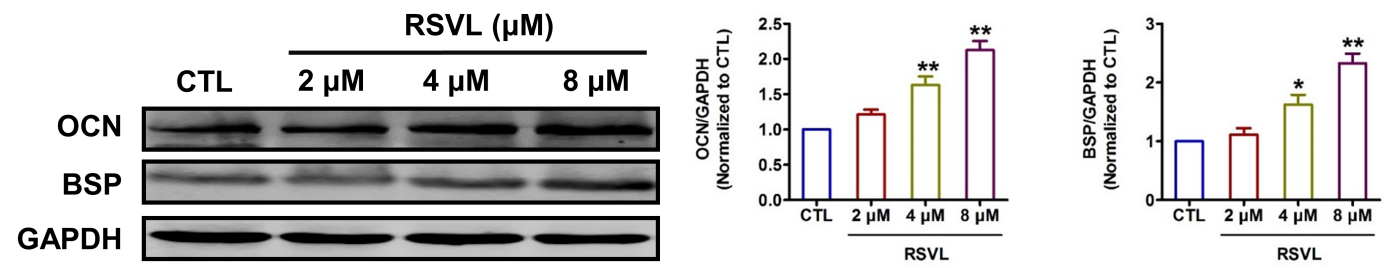

Figure 1

RSVL promoted the osteogenic differentiation of BMSCs. (A) The chemical structure of RSVL. (B) ALP staining was performed to detect the ALP activity and matrix mineralization deposition of BMSCs after RSVL treatment $(n=3) .{ }^{*} P<0.01,{ }^{\star} * * P<0.001$ versus CTL. CTL, control. (C) ARS staining was applied to measure the osteogenic differentiation of BMSCs after treatment of different concentrations of RSVL $(n=3) .{ }^{*} P<0.05, * \star * P<0.001$ versus CTL. (D) Real time $q P C R$ analysis was used to examine the expression of osteogenic differentiation-related genes of BMSCs treated with RSVL $(n=3)$. ${ }^{*}<0.05, * \star P<$ $0.01,{ }^{*} * \mathrm{P}<0.001$ versus CTL. (E) Western blot analysis was used to investigate the protein expression levels of $O C N$ and $B S P(n=3)$. ${ }^{*}<0.05,{ }^{*} P<0.01$ versus $C T L$. 

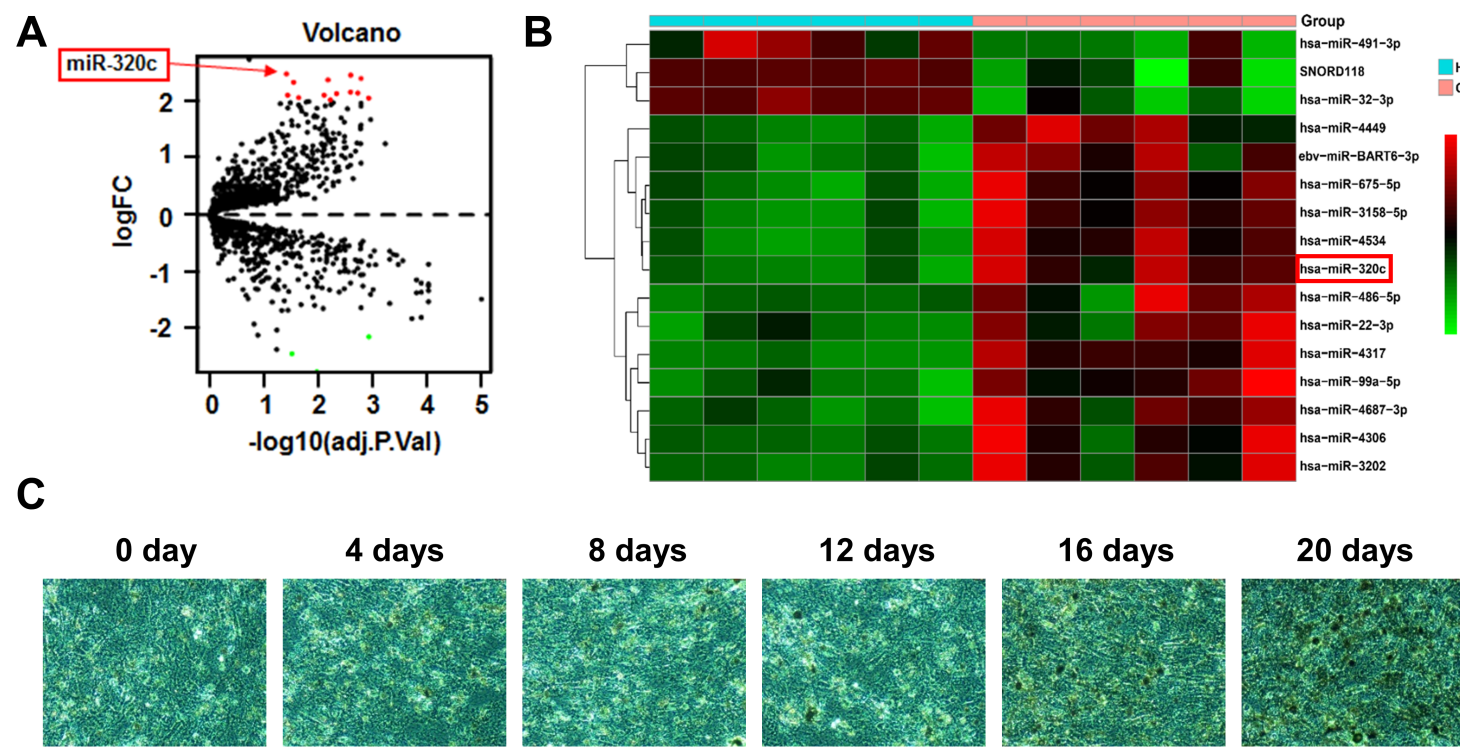

Healthy controls
Osteoporosis

C
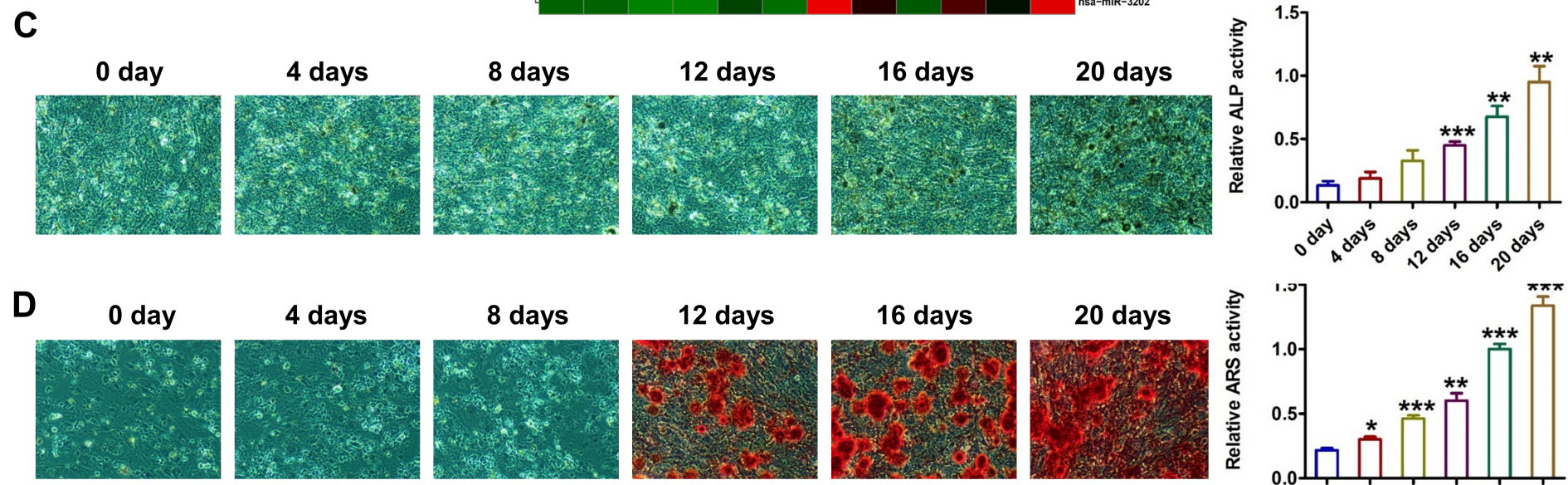

E
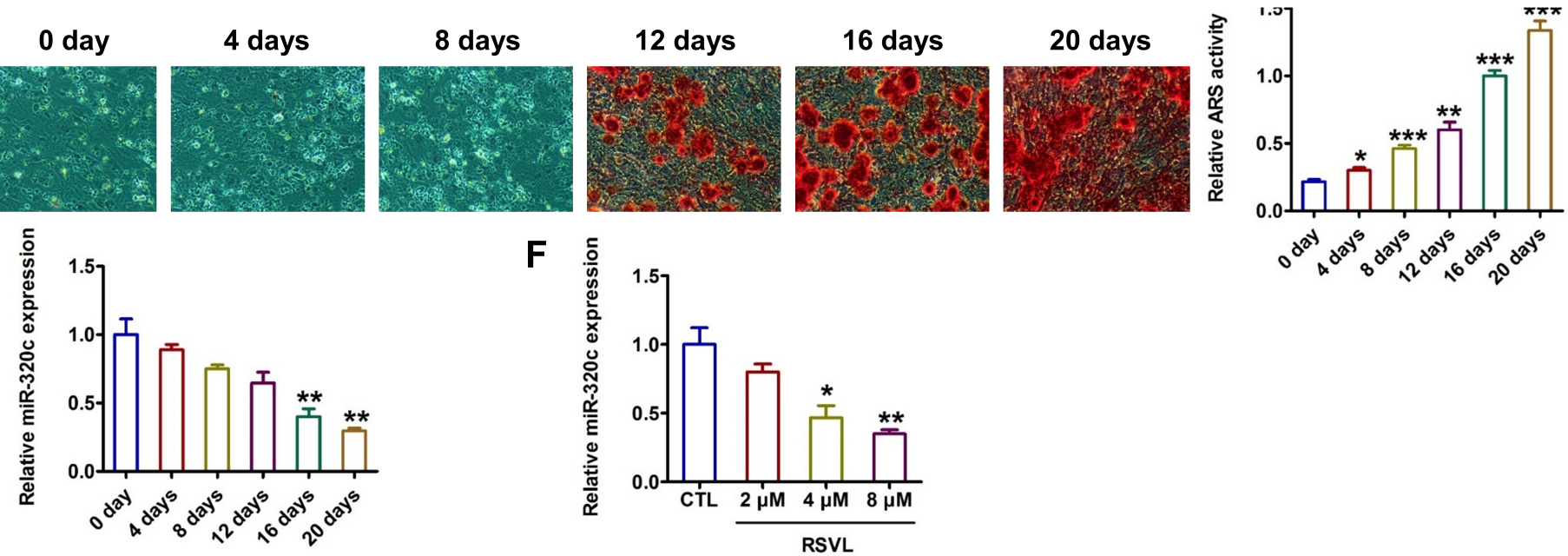

Figure 2

The expression level of miR-320c during the osteogenic differentiation and after RSVL treatment. (A and B) ALP and ARS stainings were performed to detect the osteogenic differentiation of BMSCs for different days $(n=3) .{ }^{*} P<0.05,{ }^{*} P<0.01,{ }^{* *} P<0.001$ versus $C T L$. (C) Real time $q P C R$ analysis was used to explore the expression level of miR-320c in BMSCs after osteogenic induction of different days $(n=3)$. ${ }^{*} P$ $<0.01$ versus CTL. (D) Real time qPCR analysis was used to study the expression level of miR-320c in BMSCs after treatment of RSVL $(n=3) .{ }^{*} P<0.05, * * P<0.01$ versus $C T L$. 
A

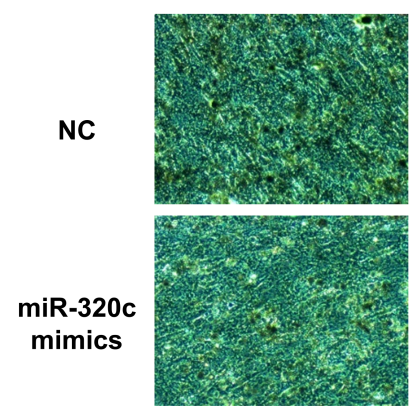

C

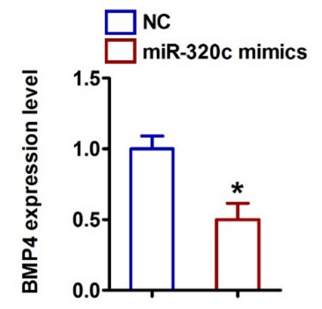

D

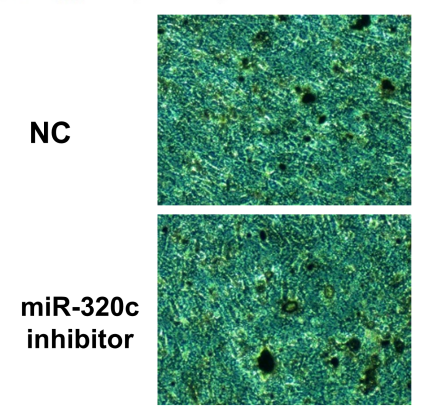

$\mathbf{F}$

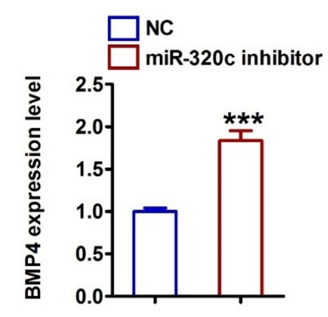

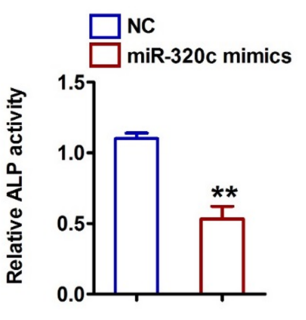

B
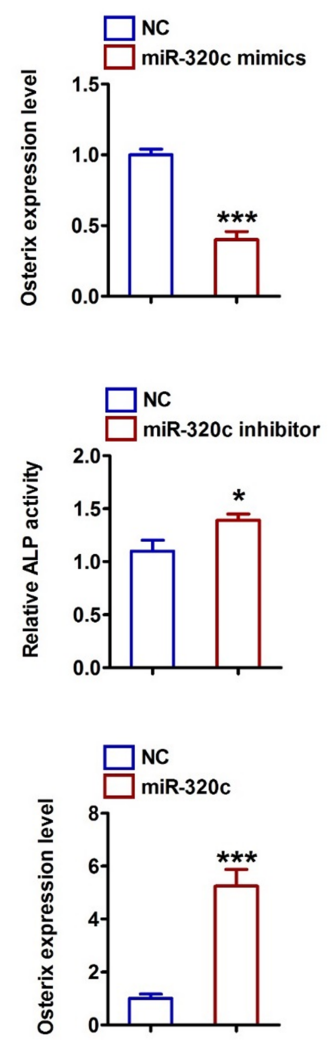

E
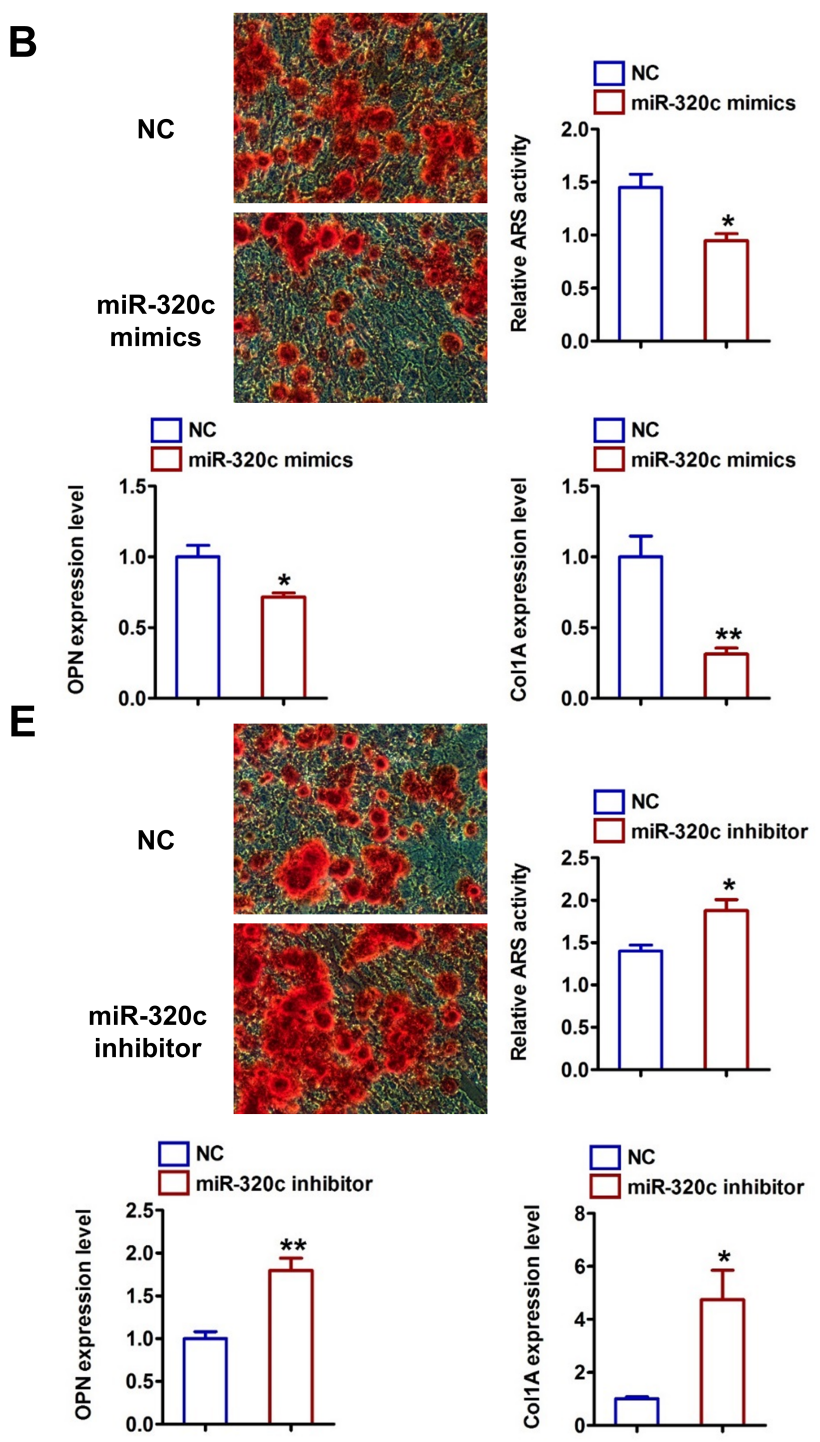

Figure 3

The effects of miR-320c on the osteogenic differentiation of BMSCs. (A and B) ALP staining (A) and ARS (B) staining showed that miR-320c mimics inhibited the osteogenic differentiation of BMSCs $(n=3)$. * $<$ 0.05 , ${ }^{*} \mathrm{P}<0.01$ versus CTL. (C) Real time qPCR analysis indicated that miR-320c mimics reduced the mRNA expression level of osteoblast-related genes $(n=3)$. ${ }^{*} P<0.05$, ${ }^{*} P<0.01,{ }^{* \star *} P<0.001$ versus CTL. (D and E) ALP and ARS staining of the osteogenic differentiation of BMSCs transfected with miR-320c inhibitor ( $n=3) .{ }^{*}<0.05$ versus CTL. (F) Real time $q P C R$ analysis of the mRNA transcripts of key osteogenesis-specific genes $(n=3)$. ${ }^{*}<0.05,{ }^{*} P<0.01,{ }^{\star *} P<0.001$ versus CTL. 
A

CTL

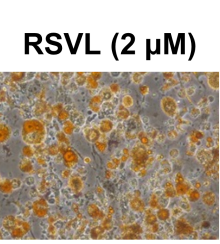

RSVL (4 $\mu \mathrm{M})$

RSVL (8 $\mu \mathrm{M})$
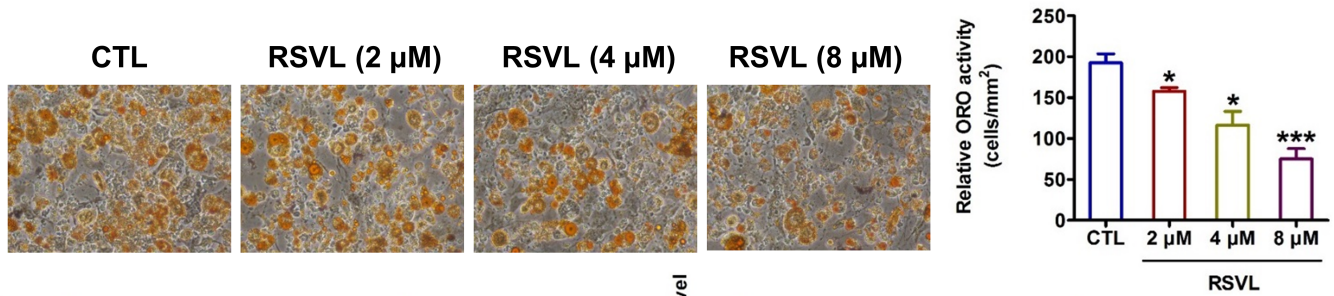

B
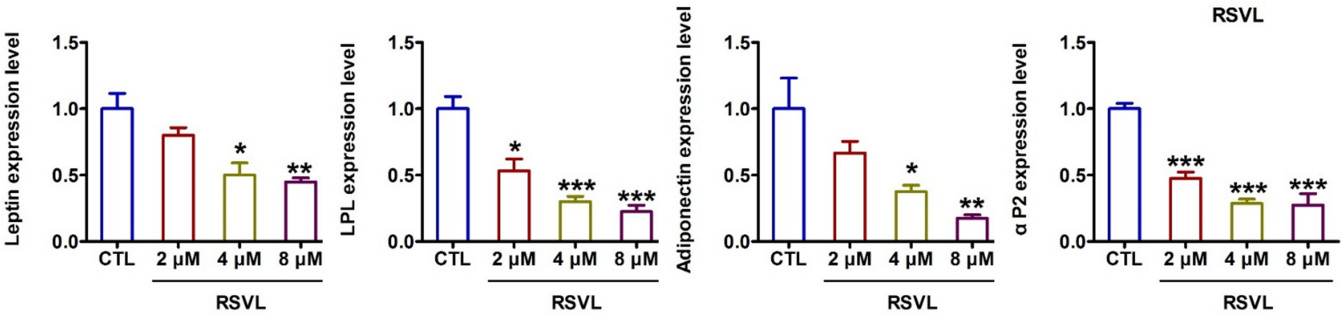

C

$\operatorname{RSVL}(\mu \mathrm{M})$

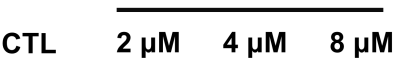

PPARY

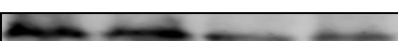

FABP4

GAPDH $\longrightarrow$
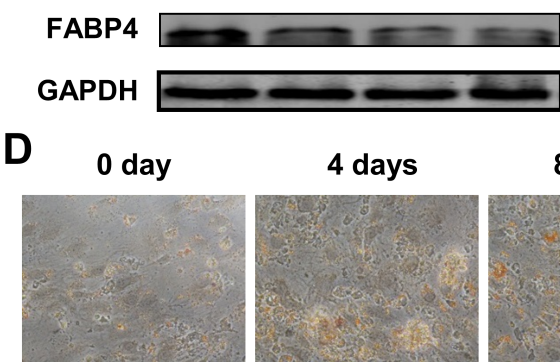

4 days
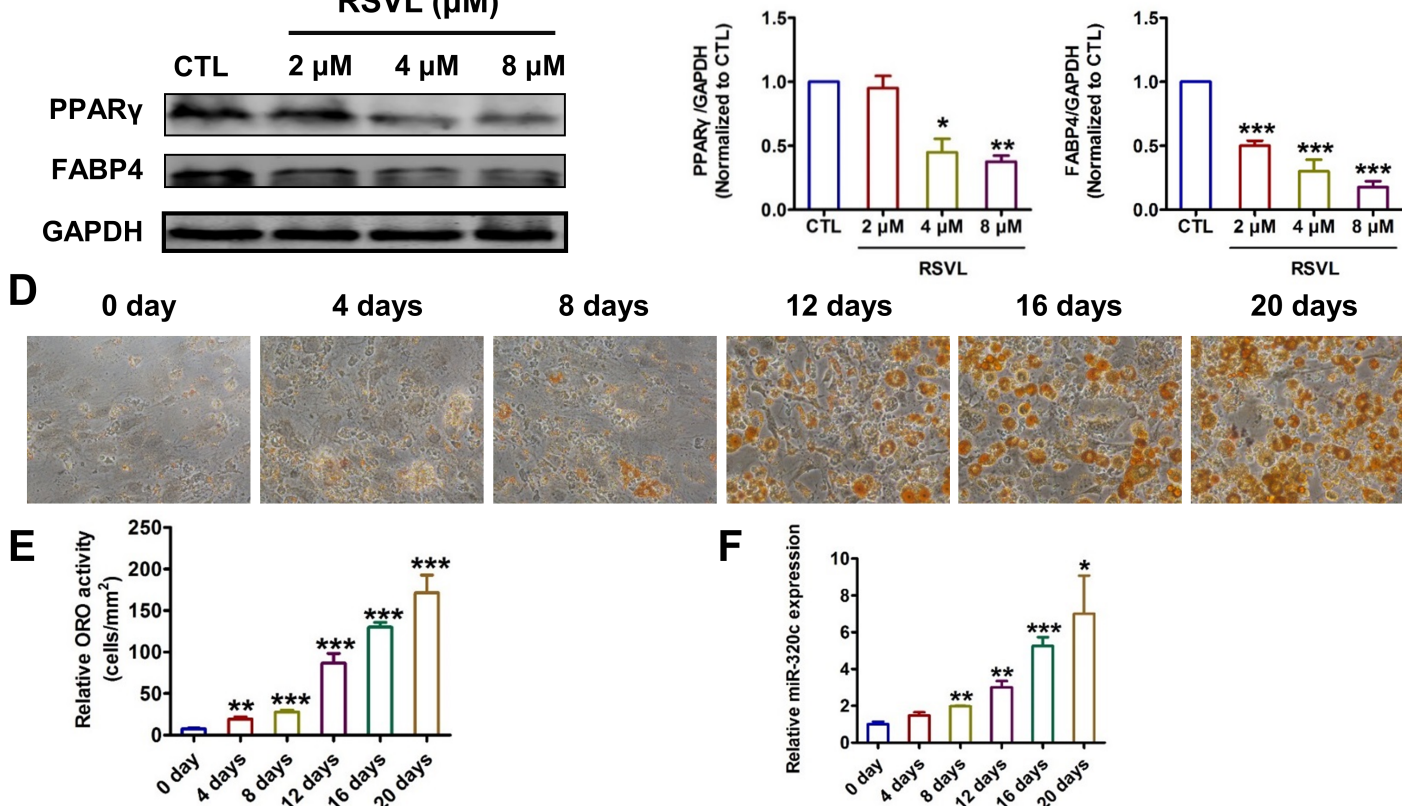

16 days

20 days
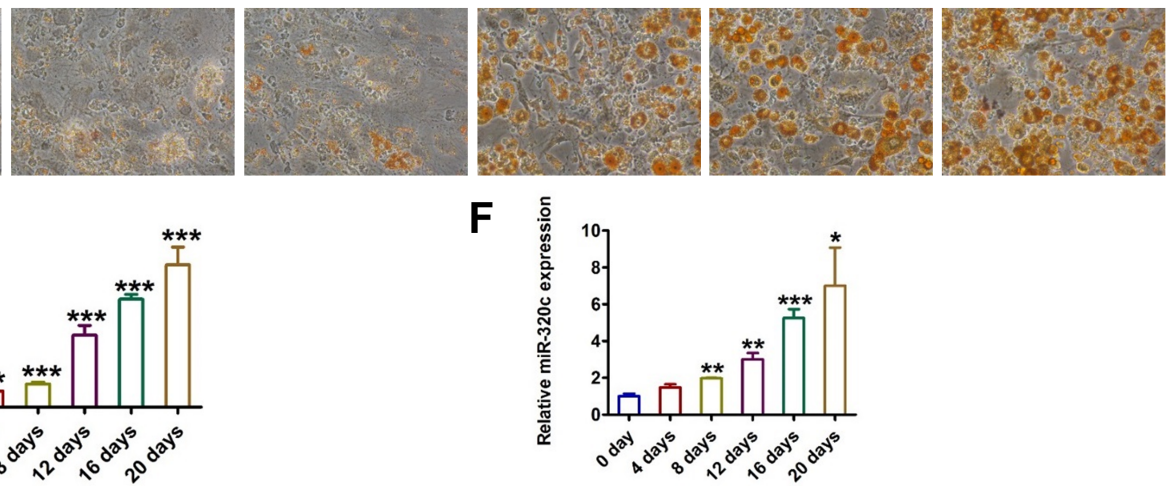

Figure 4

The roles of RSVL in the adipogenic differentiation of BMSCs. (A) RSVL treatment reduced the osteogenic differentiation of BMSCs in a dose-dependent manner $(n=3)$. ${ }^{\star} P<0.05,{ }^{\star} * * P<0.001$ versus CTL. (B) The expression level of adipocyte-related genes was reduced by the RSVL treatment $(n=3)$. ${ }^{*}<<0.05$, ${ }^{* *} P<$ $0.01,{ }^{*} * \mathrm{P}<0.001$ versus CTL. (C) The protein levels of adipogenesis-correlated genes were downregulated after RSVL treatment $(n=3)$. ${ }^{*} P<0.05$, ${ }^{*} P<0.01$, ${ }^{*} * P<0.001$ versus CTL. (D and E) ORO staining detected the number of oil droplets of BMSCs after adipogenesis of different days $(n=3)$. ${ }^{*} P<$ $0.01,{ }^{*} * \mathrm{P}<0.001$ versus CTL. (F) The expression level of miR-320c was gradually increased during the process of adipogenesis $(n=3)$. ${ }^{*} P<0.05, * * P<0.01, * \star * P<0.001$ versus CTL. 
A

NC

miR-320c

mimics
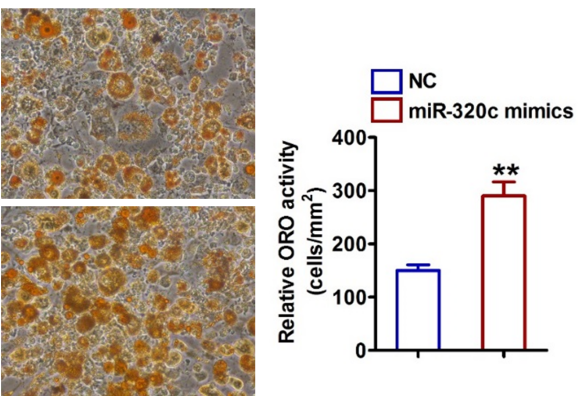

C

NC

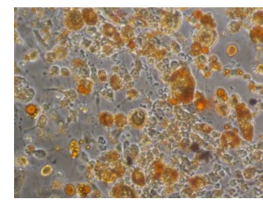

miR-320c

inhibitor
B
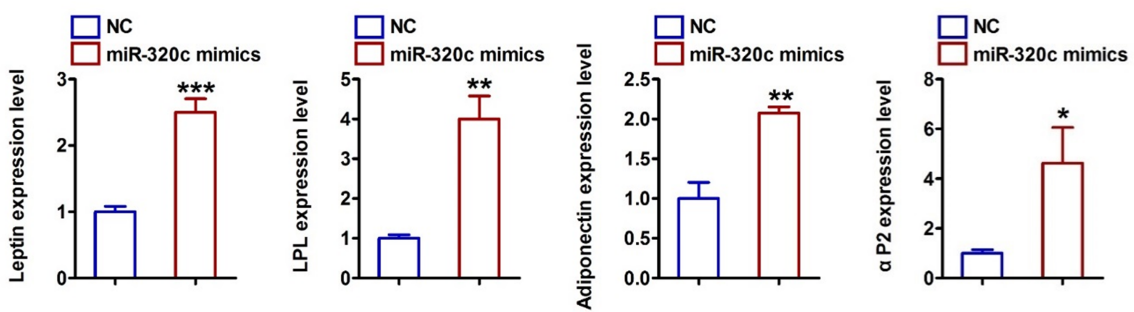

D
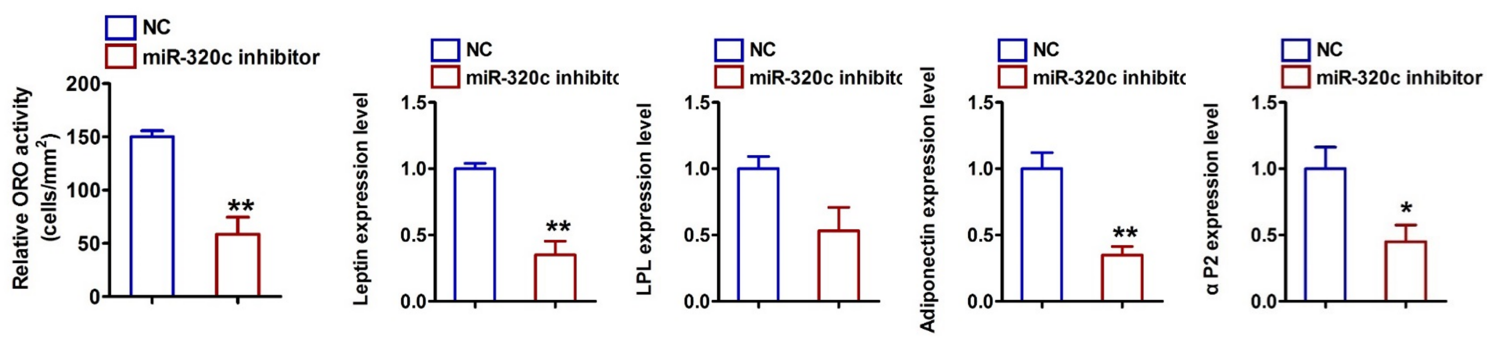

\section{Figure 5}

miR-320c played a positive role in the adipogenesis of BMSCs. (A) ORO staining of oil droplets of BMSCs after treatment of miR-320c mimics and NC $(n=3) .{ }^{*} P<0.01$ versus CTL. (B) Quantitative RT-PCR analysis of adipogenesis-differentiated genes in BMSCs after transfection of miR-320c mimics or NC $(n=3) .{ }^{*} P<0.05,{ }^{*} P<0.01, * \star * P<0.001$ versus CTL. (C) Knockdown of miR-320c inhibited the adipogenesis of BMSCs compared with $N C(n=3) .{ }^{*} P<0.01$ versus CTL. (D) Real-time qPCR analysis of leptin, lipoprotein lipase (LPL), adiponectin, and adipocyte protein 2 (aP2) in BMSCs with miR-320c knockdown or $\mathrm{NC}(\mathrm{n}=3)$. ${ }^{*} \mathrm{P}<0.05,{ }^{*} \mathrm{P}<0.01$ versus $\mathrm{CTL}$. 
A
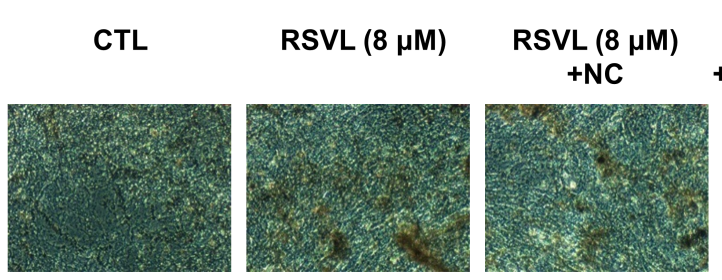

RSVL (8 $\mu \mathrm{M})$

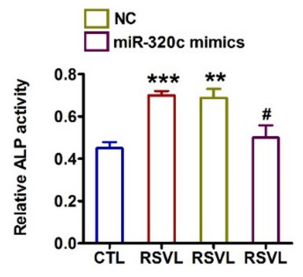

CTL

RSVL (8 $\mu \mathrm{M})$

RSVL (8 $\mu \mathrm{M})$

$+\mathrm{NC}$

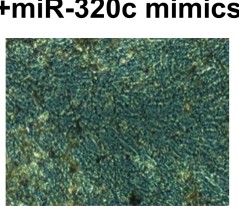

RSVL $(8 \mu \mathrm{M})$

$\square \mathrm{NC}$

+ miR-320c mimics

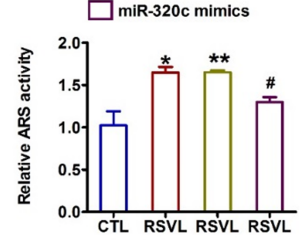

C
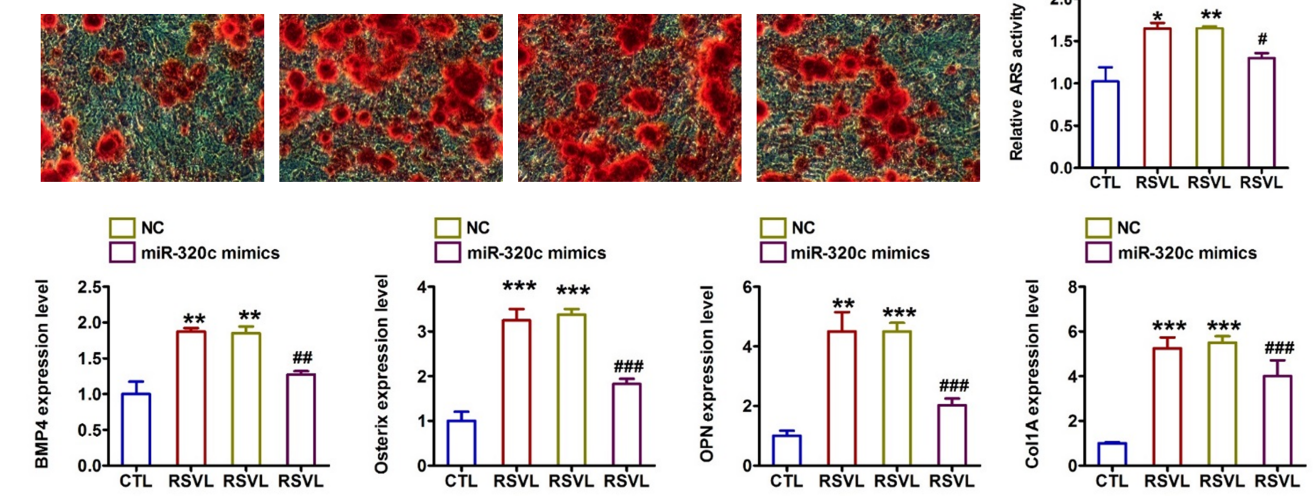

D

CTL

RSVL (8 $\mu \mathrm{M})$

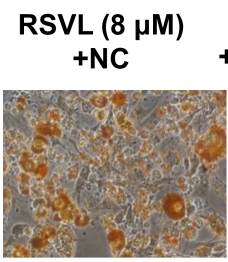

RSVL $(8 \mu \mathrm{M})$ +miR-320c mimics

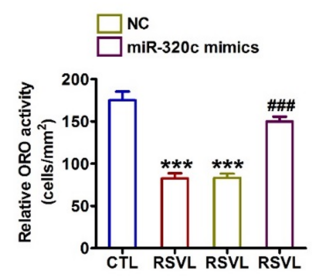

E
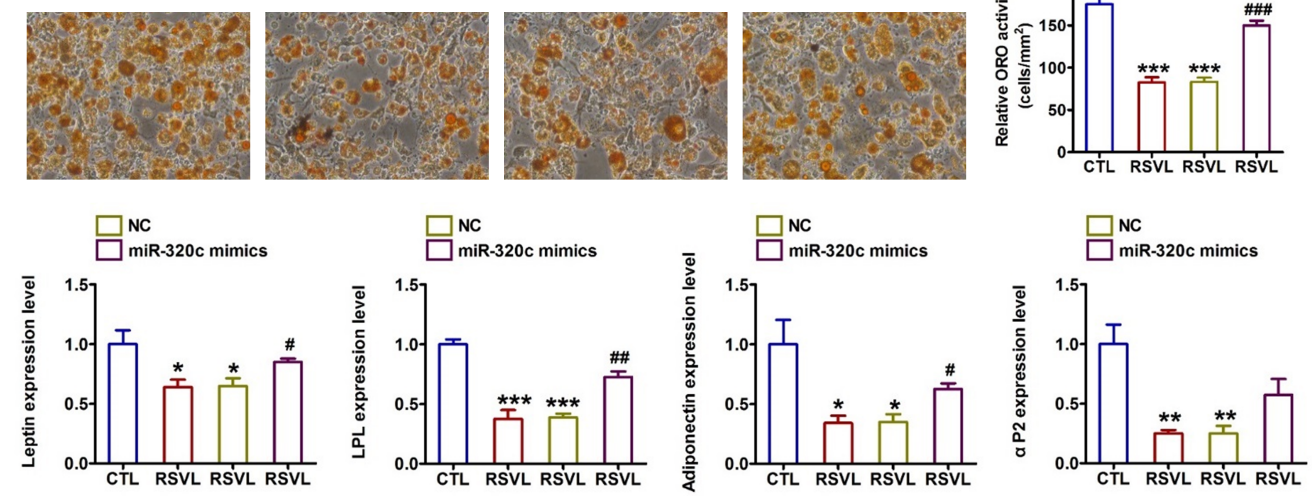

Figure 6

miR-320c regulated RSVL-promoted osteogenic differentiation and RSVL-suppressed adipogenic differentiation of BMSCs. (A and B) Overexpression of miR-320c using miR-320c mimics significantly inhibited the osteogenesis in RSVL-treated BMSCs $(n=3)$. ${ }^{*} P<0.05,{ }^{*} P<0.01$, ${ }^{\star \star *} P<0.001$ versus $C T L$. $\# P<0.05$ versus RSVL. (C) Knockout of miR-320c markedly reduced the mRNA levels of BMP4, Osterix, OPN, Col1 A in RSVL-treated BMSCs $(n=3)$. ${ }^{\star *} P<0.01,{ }^{\star \star *} \mathrm{P}<0.001$ versus CTL. \#\#P $<0.01$, \#\#\#P<0.001 versus RSVL. (D) miR-320c mimics observably increased the number of adipocytes, which was inhibited by RSVL treatment $(n=3)$. ${ }^{\star *} P \mathrm{P}<0.001$ versus CTL. \#\#\#P $<0.001$ versus RSVL. (E) The mRNA expression levels of adipocyte-related genes were upregulated by miR-320c mimics, which was downregulated by RSVL treatment $(n=3) .{ }^{*} P<0.05,{ }^{\star} * P<0.01,{ }^{\star} * \star P<0.001$ versus $C T L$. $\# P<0.05$, \#P $<0.01$ versus RSVL. 


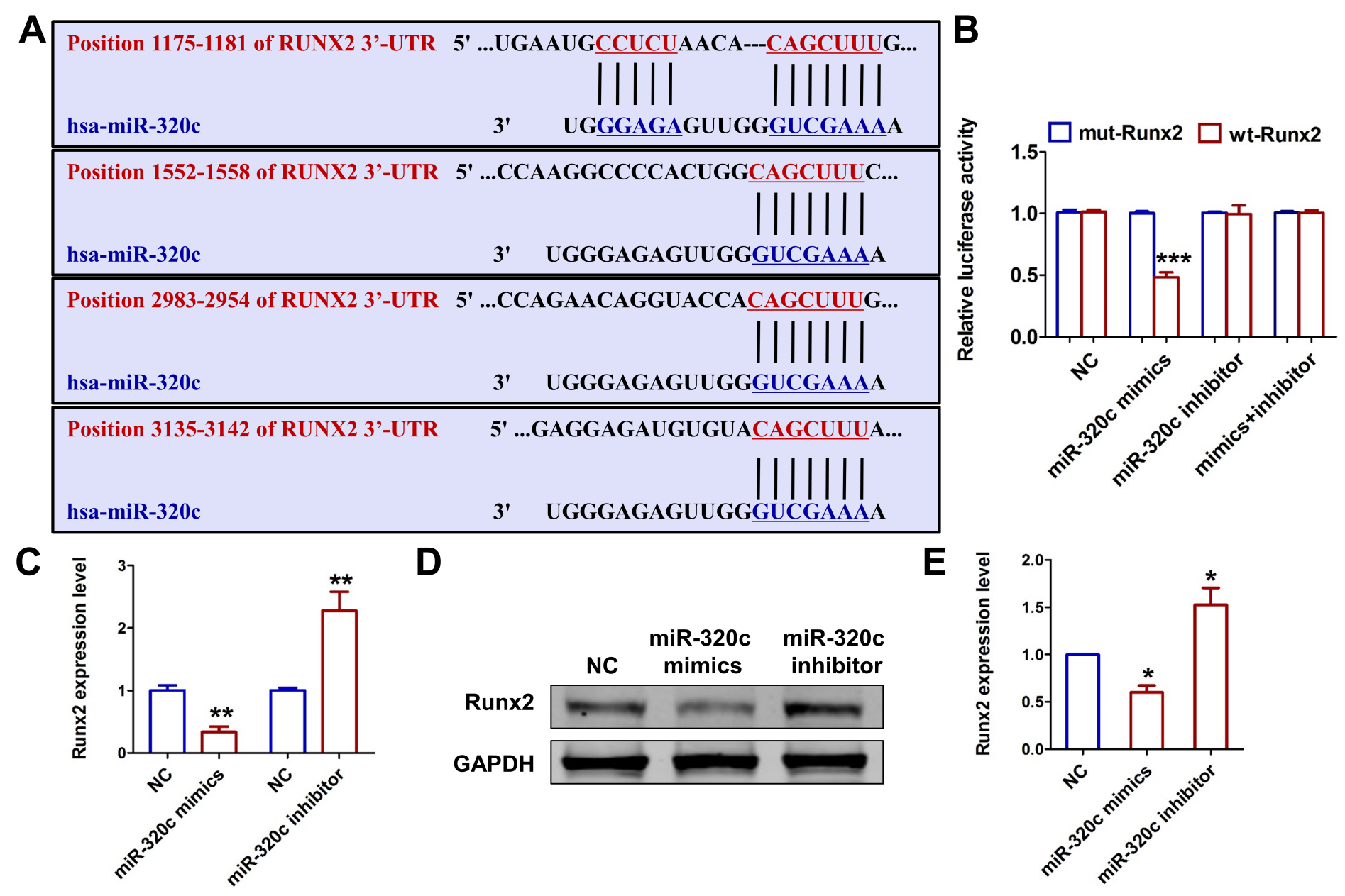

Figure 7

miR-320c regulated the osteogenic differentiation of BMSCs by targeting Runx2. (A) The possible binding sequence was predicted using TargetScan Human 7.2. (B) Luciferase assay was conducted to detect the interaction between miR-320c and Runx2. WT, wild type. MUT, mutant type. (C-E) Real time qPCR and western blot analysis were applied to detect the protein levels of Runx2 $(n=3)$. ${ }^{*}<<0.05,{ }^{*} P<0.01$ versus CTL. 